\title{
Analysis on the Effects of the Improved Power Forecast System of Zhong San Jia Wind Power Plant in Liaoning Province of China
}

\author{
Chao Zhang ${ }^{1, \text { a }}$ \\ ${ }^{1}$ Datang Renewable Power Test and Research Institute, Beijing, China \\ a93838142@qq.com
}

Keywords: power forecast; relative error; the year of 2013.

\begin{abstract}
This article introduces the error comparison of the improved wind power forecast system which changes the data input and re-models and the original one, which was done in Liaoning Zhong San Jia Wind Power Plant. Throughout one year's contrast analysis in 2003, it is found that the accuracy rate has increased by 6 per cent in the strong wind months, about 1.5 per cent in the weak wind months, and 4 per cent in the whole year. All the data shows that the improved system is excellent.
\end{abstract}

\section{Introduction}

A satisfactory wind power forecasting system is necessary for grid connection in wind farms. Currently, the wind power systems installed in wind farms are original from several software companies. However, the development and operation interfaces of these systems are different, which causes great inconvenience for our company. More importantly, each wind farm only has the client of its wind power system. Therefore, our company cannot independently upgrade the system and develop much more softwares that are consistent with our company's current situation.

Wind power forecasting system can be roughly divided into two parts: pre-treatment section with meteorological modeling and data forecasting which can measure the wind speed and forecast the wind direction; post-treatment section in which the fan power can be calculated according to wind speed and direction. If the accurate results can be measured in the pre-treatment, it will be greatly helpful to forecast the power.

Our company has been cooperating with Peking University since 2013. By introducing the model of mode output statistics and 4D data assimilation system, we could construct weather model and greatly improve the accuracy of wind speed and direction measurement, which has been tested in the three wind farms of our company in Liaoning Province.

\section{Research Contents and Implementing Scheme}

The research contents of this project includes: (1) research on numerical weather prediction technology aimed at the wind power forecast; (2) research on the physical and statistics methods of calculating generating capacity; (3) research on the construction project of power forecast system

It provides the wind field requested by the project and the forecast of other meteorological factors by setting up high resolution mesoscale numerical forecasting model system. The system is based on the mesoscale regional model of WRF (Weather Research and Forecasting) and the data analysis assimilation system of ADAS (ARPS Data Analysis System). It takes GFS (Global Forecasting System) Analysis Field as the initial guess field and uses the ADAS model to assimilates kinds of observation data such as SYNOP, SHIP, BUOY, METAR, AMDAR, FY2C-IR1\&VIS, CINRAD (SA,SB), and AWS, to obtain the high resolution sophistication initial field needed by the WRF model and generate the lateral boundary conditions. All kinds of the meteorological forecast fields can be obtained according to the integral of the WRF model.

The calculation methods of electric-power output, including physical method, statistical method and integrated method, all have advantages and disadvantages. Physical method does not require large amounts of measurement data, but requires the accurate mathematical description of physical 
properties and atmospheric characteristics. These equations are difficult to solve and require massive computational information and long computing time. Statistical method does not solve physical equations and its calculating speed is faster. But it requires a lot of historical and actual data. Currently, our country has built the weather data collection system of real-time micropower in wind farms, which can meet the needs of wind power prediction. Comprehensive method requires numerical intermediate products and weather forecast data. The forecast period is short, but it is an attempt to make prediction.

The construction scheme of the system is a set of systems engineering since it concerns the collection of data and the construction of real-time wind monitoring system, numeral center, as well as network software system.

Research on the Numerical Weather Prediction System. The height $(60-100 \mathrm{~m})$ of wind power generation tower mainly lies in the near-surface layer. However, the wind speed changes a lot in the vertical direction and regular interpolation method can't be used to calculate the subtle change of wind field within 100 meters near the ground. Therefore, we should take advantage of the momentum of ground, heat, water vapor flux forecasted in the process of model integral, the roughness of the position of the wind power, and so on, to calculate the near-surface wind speed through related physical laws. The project uses the near-surface layer wind speed diagnosis analysis technology put up by Geleyn, which functions many years in the top global weather center-ECWMF and is an effective way of forecasting near-surface wind.

The model rings twice every day (8:00 and 20:00 at Beijing time ) and does the about 130-minute parallel computation on the high-performance computer. At present, it takes every minute to judge the data and get information. After the needed information is obtained, the model will start.

The horizontal resolution of the model is $9 \mathrm{~km}$ and the horizontal range is $2376 \mathrm{~km} * 2376 \mathrm{~km}$. The vertical direction of the model is 31 layers. The cumulus parameterization scheme is not used in the model, and the WRF Single-Moment 6-class Scheme suitable for the high-resolution simulation is chosen as the microphysical scheme, which includes the ice phase physical process of ice, snow, graupel, and so on. The long-wave radiation scheme is Rapid Radiative Transfer Model, and Dudhia Scheme is chosen as the short-wave radiation scheme. The land surface scheme is Noah Land Model. It divides the land into 4 layers and considers the temperature and humidity of land. The snow cap changes from $0.0 \sim 1.0$ and the frozen earth can be considered in the physical process. The near-surface layer scheme is MM5 Similarity Scheme and Yonsei University Scheme is chosen as the boundary layer scheme. Non-local K Scheme is adopted in the entrainment zone to do the display resolution, and the $\mathrm{K}$ profile line of the instable mixing layer shows paradoxical distribution.

Research on the Physical and Statistics Methods of Calculating Generating Capacity. The neural network is a kind of parallel processing connecting network which shows the basic functions of human's brain. It is based on the research on the way human thinks then simplify, abstract, and simulate in the mathematic method. A neural network is made up of many interlinked neurons, which are the basic processing unit of neural network and the simplification and simulation of biological neurons. The picture 2-7-1 shows a simplified neuron structure, which is a multiple- input, one-output nonlinear element and its input/output relationship can be described as

$$
\left\{\begin{array}{l}
I=\sum_{j=1}^{n} w_{j} x_{j}-\theta \\
y=f(I)
\end{array} .\right.
$$

The "xj $(j=1,2, \cdots, n) "$ is the input signal from other cells, and " $\theta$ " is the threshold value. Weigh coefficient "wj" represents the intensity of connection and shows the load of synapse. The "f(x)", which describes the transfer characteristic of biological neurons, is called excitation function or transfer function. Its basic function is : (1) control the activation of input to output; (2) do the function transformation of input to output; (3) change the input of possible infinite field into the output of specified limited range 
The BP neural network is a kind of multilayer feedforward neural network. The adopted adjustment rule whose name is from network weights is Backward Propogation Method (BP Method), namely, BP Method. It is put up in 1986 by Rumelhart and others. Henceforward the BP neural network has been widely applied. Statistics show that $80 \%$ to $90 \%$ of the neural network models use BP network or its versions. The BP network is the core part of feedforward network and it reflects the most golden and perfect content of the neural network.

\section{Condition of the Tested Wind Plant}

The tested wind plant is Zhong San Jia Wind Power Plant of Datang Renewable Power Zhaoyang Corporation in Liaoning Province. There are 20 goldwind wind turbines of $1500 \mathrm{~kW}$, which are grid-connected in February of 2010. And the picture below shows its condition of limiting power.

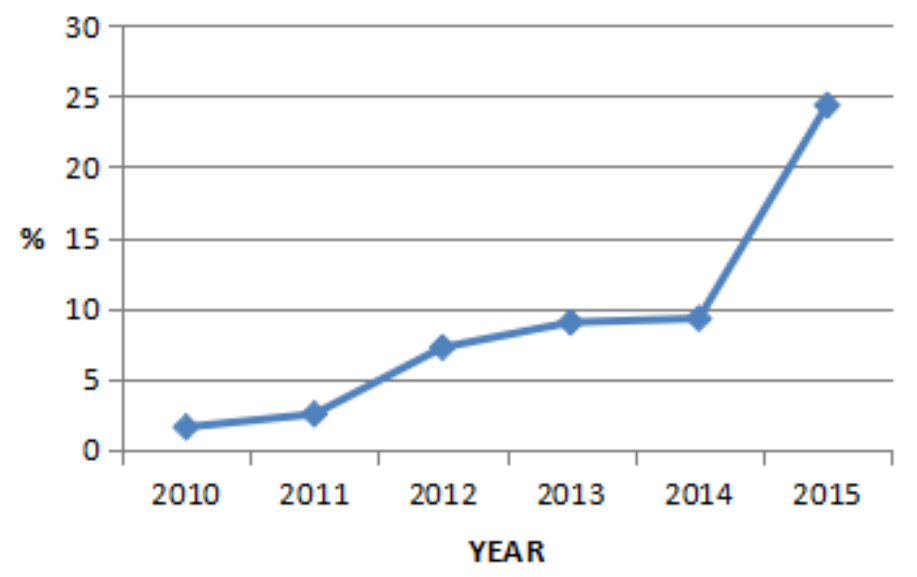

Figure 1 The Annual Condition of Limiting Power in Zhong San Jia Wind Power Plant

It can be seen that the condition of limiting power in this wind power plant is increasingly serious. At present, the power network dispatching in Liaoning province adopts the system of power prediction comparison and preliminary assessment. It determines the amount of liming power according to the level of power forecast in every wind power plant. The improved power forecast scheme has much practical significance in Zhong San Jia Wind Power Plant.

The prevailing wind direction of Zhong San Jia Wind Power Plant is north and north-north-east. As to the wind turbines layout, there are 6 wind turbines on a ridge of the west-north direction and other 14 wind turbines on another ridge of the east-south direction. The template machines are the No.2 and No.15 wind turbines, which are not limited by the power.

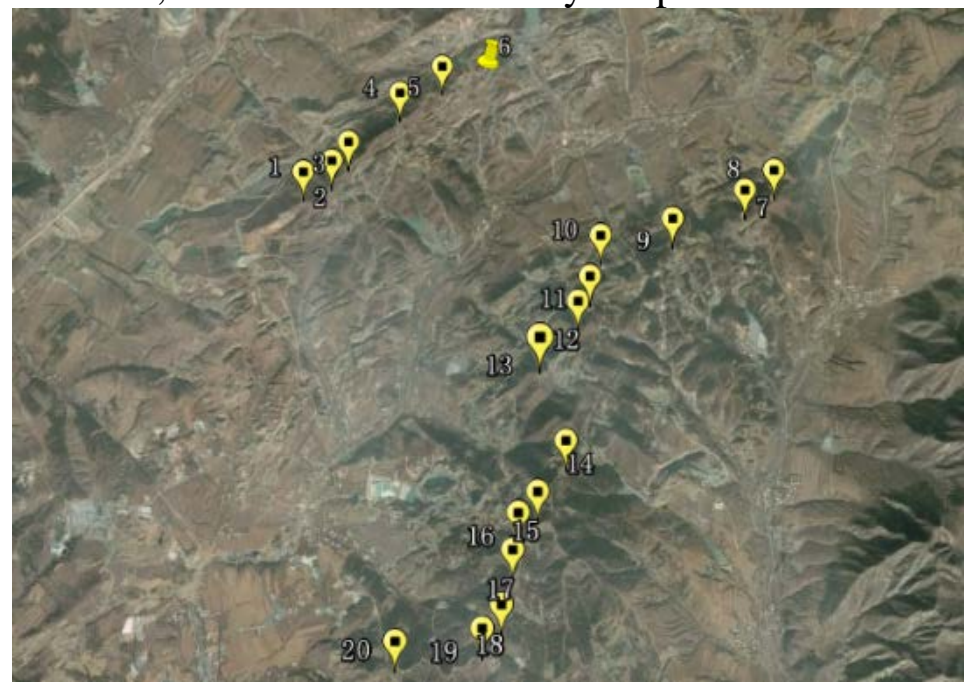

Figure 2 The Schematic Diagram of the Wind Turbines Layout in Zhong San Jia Wind Power Plant 


\section{Assessment Indexes}

According to The Enforcement Regulation of the Management of the Grid-connection Operation of Power Plants in the Northeastern Region and The Enforcement Regulation of the Management Way of Grid-connected Power Plants in the Northeastern Region Providing Auxiliary Service, as well as the regulations of The Notification on Carrying out the Work of Reselecting the Template Machines in the Wind Power Plant issued by Niaoning National Electric Power Dispatching Center, the assessment standards are as follows.

When it is calculated by the day on the basis of background data, the daily deviation rate of the fitting full-field generating capacity of template machines to the real daily generating capacity is no more than 3 per cent.

When it is calculated by the day on the basis of background data, the max time deviation rate of the fitting full-field generating capacity of template machines in single time period to the real generating capacity is no more than 10 per cent. The formula is : " the daily deviation rate = (the real daily generating capacity-the fitting generating capacity of template machines) / the real daily generating capacity, the max time deviation rate = MAX (time deviation rate $\mathrm{i},(\mathrm{i}=1,2,3 \cdots 96)) "$.

The root-mean-square error (RMSE) is no less than 85 per cent. Its formula is

$$
R M S=\sqrt{\frac{\sum_{i=1}^{N}\left(P_{\text {predicted }}-P_{\text {real }}\right)^{2}}{N}}
$$<smiles>[R10]C#CC(C)(C)C</smiles>

\section{Collection of the Field Data}

The core of the optimization of power prediction is data which include the historical data of power generation in the wind power plant and the historical data of power prediction in the original system. In October of 2013, after confirming the wind power plant as the project site, we immediately set up the project team and successively went to Niaoning province to copy the historical data of several wind power plants like Sanjia, Shuangmiao, Xiao tazi, and so on, to let related staff of Peking University do the modeling analysis. Through the simple data cleaning in the early stage, we found the data copied from Zhong San Jia is the most complete. In order to do the comparison more conveniently and ensure the validity of optimization method, we chose Zhong San Jia as the experimental site to do the power optimization test. Meanwhile, we did the production and extract of meteorological data according to the meteorological data information provided by Peking University. In July of 2014, the new resource company put up new request for the analysis of power optimization, which is lengthening the time interval of verifying the validity of optimization method. Therefore, we went to the site again to copy the historical data of Zhong San Jia and Shuangmiao wind power plant. After the data processing and analysis, we finally set the model parameter and put the prediction on the wind power prediction platform to do the graphical representation.

\section{Collections of Meteorological Data}

Meteorological data is the basic requirement of power prediction, which requires more predictive accuracy. Therefore, we establish the refinement local meteorological model and introduce Model Output Statistics (MOS) and Weather Research Forecast Four-Dimensional Data Assimilation (WRF FDDA), which finally contributes to the meteorological element information of each fan.

Localization of Meteorological Model. We chose the parameters of meteorological model according to local actual condition, so as to make the model most truly reflect the local climate conditions and reduce the prediction errors of mesoscale model. The parameters consist of :

radiation parameterization 
microphysics process parameterization

boundary-layer parameterization scheme (Wind-driven generator mainly lies in the atmospheric boundary layer, so the effect of different boundary-layer parameterization schemes on the forecast ability is one of the factors which the scheme considers )

cumulus convective parameters

parameterization of land surface process

At present, the ability of many parameterization schemes to describe the meso-and micro-scale system is insufficient and the effect of mode in the district forecast and the adaptability of parameterization schemes are very important, so it is of much significance to develop the parameterization scheme which is suitable for local features.

The Adjustment of MOS Automatic Model. The recorded field data based MOS system which is initiated in the whole nation is different from the general MOS system. This lies in that it can realize the online correction and can be aimed at the distribution feature of system deviation to change as the season and weather change. It replies the characteristic of error tendency with changing correction factor.

MOS system is the method which does the online correction to the system deviation of the output of meteorological mode according to the testing results. Its premise is accumulating measured value.

WRF FDDA Realizing the Localization of Meteorological Data. The numerical value assimilation method based on the data of wind field is created first in the nation. At present, all the assimilation technologies adopted by the power prediction system of the country are based on the data introduced computing model of the meteorological station. They don't assimilate the data of wind plants (use recorded field data only in the short-term prediction not in the meteorological mode) and lack the description of local weather characteristic in wind plants. This method will overcome the obstacle and solve the representative problems of daily meteorological mode and improve the prediction result by a large margin.

WRF FDDA technology is a kind of method which applies the recorded field value into the calculating process. It can improve the richness degree of the meteorological data of the initial field and eliminate the insufficiency that the original large-scale data cannot represent the local wind field well.

\section{Data Processing}

Data Pre-processing. The copied data from Zhong San Jia Wind Power Plan include the SCADA data of wind turbines and the power prediction data of the original system. They are memorized separately in the forms of goldwind formatted file and excel file. Therefore, the primary task of data modeling is to transform the goldwind wind turbine data into the form which can be read out by the analysis software and record the needed data into the database to read and analyze at any time.

Model construction. Based on the previous experience in power optimization, we adopted two approaches to construct models, table checking method and neural network method.

Table checking method is based on the original fan power curve. It uses the wind speed predicted by Ministry of Atmosphere and forecast the short-term power.

Neural network method takes all the meteorological elements into consideration, which is the input of neural network. The standard power of wind farm is the output, which helps to establish the model.

The modeling of single fan has been one of the advantages of power prediction made by Peking University. Depending on the specific circumstances, each fan can be individually modeled. It can predict the power statue of single wind turbine, which increases the flexibility and accuracy of power prediction. Therefore, it is also used in this project.

After the comparative analysis of two methods, we found that neural network method can significantly improve the power forecast accuracy. Therefore, the final built model is neural network model, and two analytical reports were written. 


\section{Results Analysis}

The author chose the data of the whole year 2013 to do the contrast analysis of the original system, actual value, as well as the improved system .

\begin{tabular}{|l|c|c|c|c|c|c|c|c|c|c|c|c|}
\hline \multicolumn{10}{|c|}{ YEAR } & \multicolumn{10}{|c|}{2013} \\
\hline MONTH & 1 & 2 & 3 & 4 & 5 & 6 & 7 & 8 & 9 & 10 & 11 & 12 \\
\hline $\begin{array}{l}\text { Evaluation } \\
\text { Data }\end{array}$ & 2976 & 2688 & 2976 & 2880 & 2976 & 2880 & 2976 & 2976 & 2880 & 2976 & 2880 & 2976 \\
\hline $\begin{array}{l}\text { Measured } \\
\text { Data }\end{array}$ & 2902 & 2683 & 2976 & 2880 & 2976 & 2880 & 2864 & 2976 & 2880 & 2976 & 2798 & 2882 \\
\hline $\begin{array}{l}\text { Jucture } \\
\text { Rating }\end{array}$ & 97.5 & 99.8 & 100 & 100 & 100 & 100 & 96.2 & 100 & 100 & 100 & 97.2 & 96.8 \\
\hline
\end{tabular}

Figure 3 The Report on the Integrity Rate of the Short-term Data Prediction in 2013

It can be seen in Figure 3 that in 2013, the original prediction system of Zhong San Jia Wind Power Plant forecasted completely in the months of March, April, May, June, August, September, October and so on. However, the forecast lacked much in January and November.

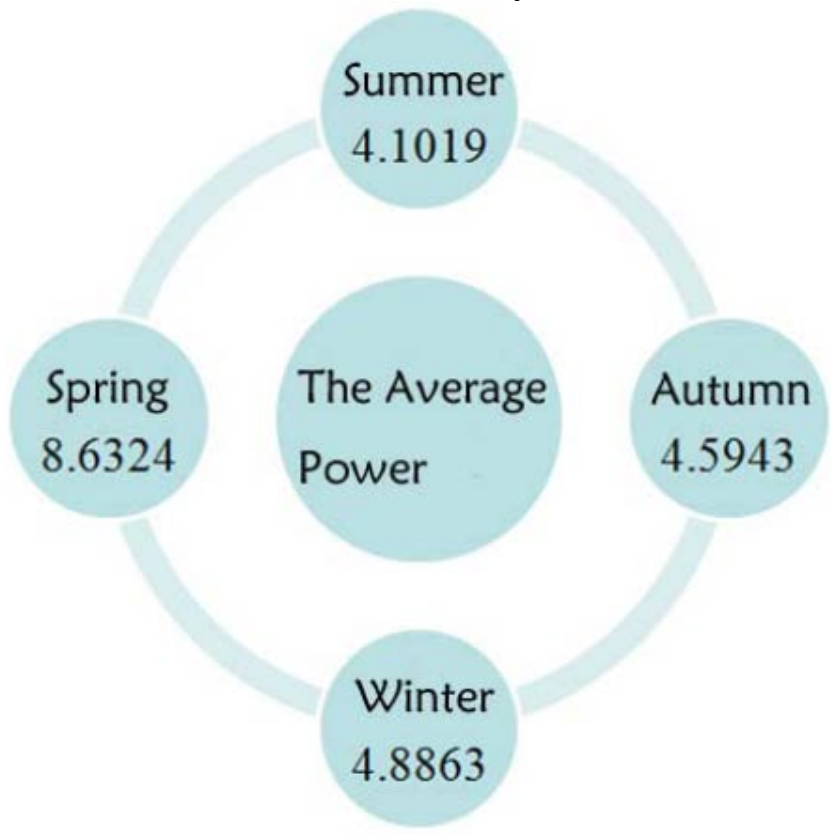

Figure 4 The Average Condition of the Real Daily Power Rate of Seasons in 2013

It can be seen in Picture 4 that in 2013, the power rate in Spring is large in Zhong San Jia Wind Power Plant. The months of March, April, May are also the most profitable. At this time, the degree of accuracy of power forecast is very important for the wind power plant.

Therefore, we distinguish the strong wind season (spring) from the weak wind season (summer and autumn) and compare the error conditions of the power forecast before and after the improvement. 


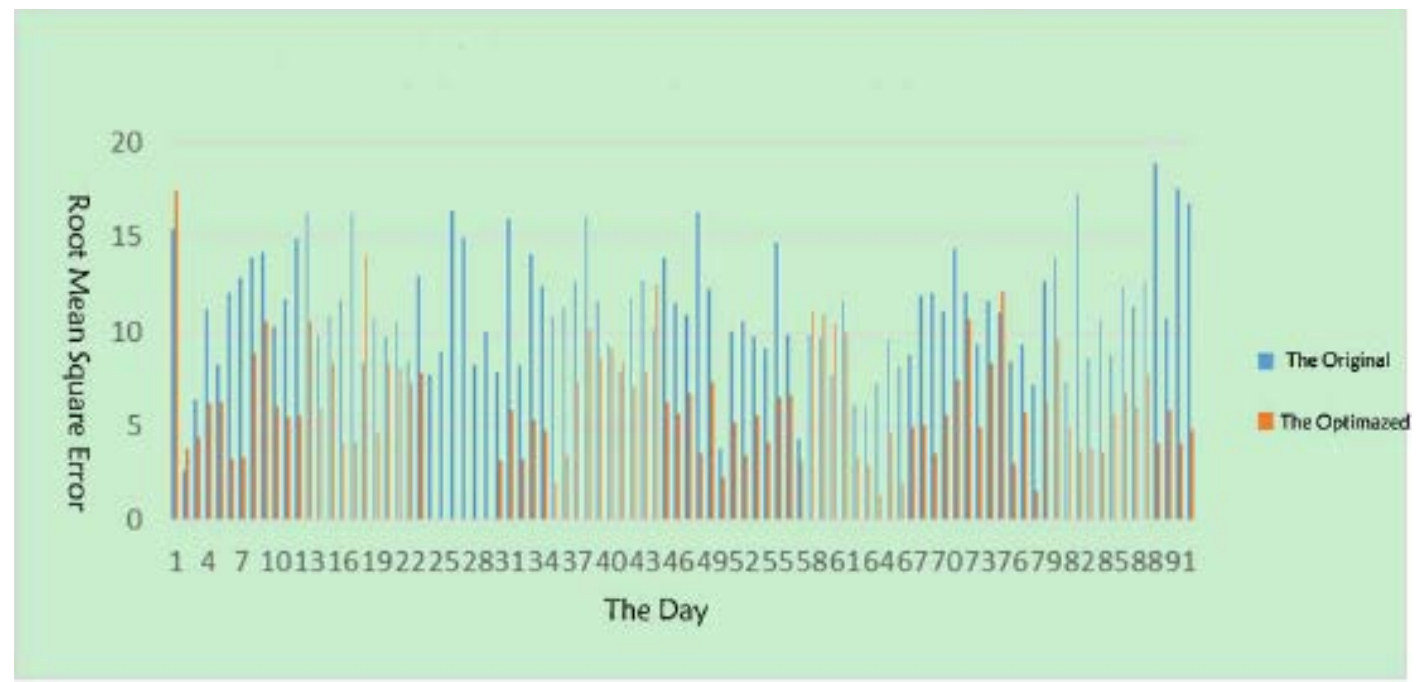

Figure 5 The Comparison of Conditions of Power Forecast before and after the Improvement in the Strong Wind Months (Spring)

Figure 5 shows that after the optimization, the number of examined days of spring decreases from 44 to 26, the number of examined days decreases by a large margin, and the whole root-mean-square error decreases obviously. Meanwhile, the average daily accuracy rate increases by 6 per cent.

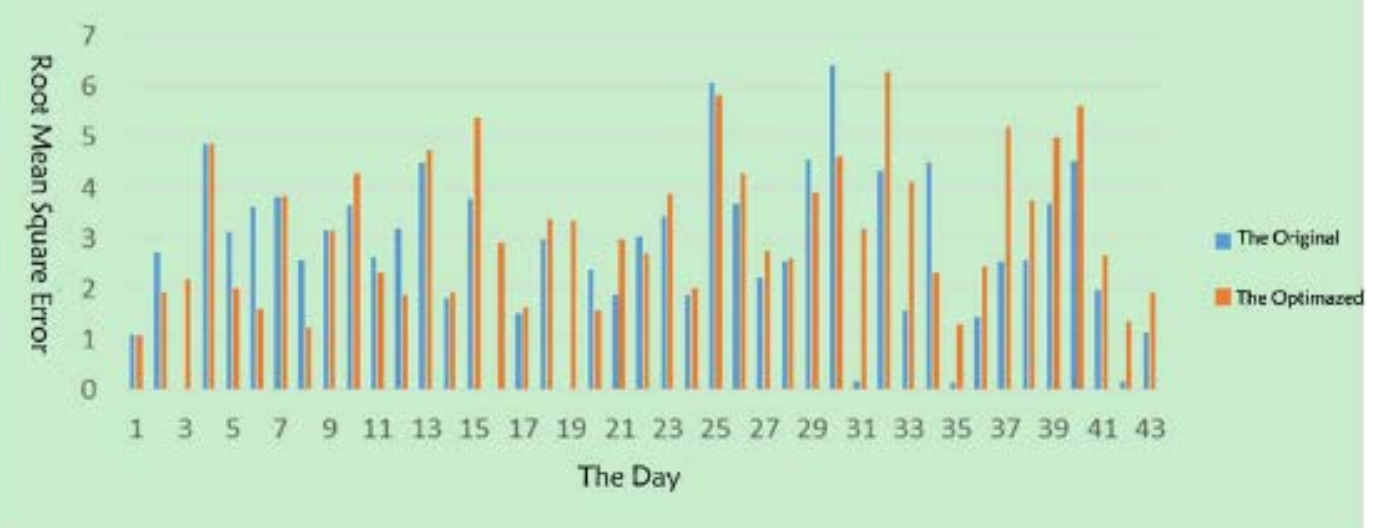

Figure 6 The Comparison of Conditions of Power Forecast before and after the Improvement in the Weak Wind Months (Summer and Autumn)

In the weak wind months, after the optimization, the whole root-mean-square error decreases a little and the average daily accuracy rate increases from the original $88.56 \%$ to the present $90 \%$.

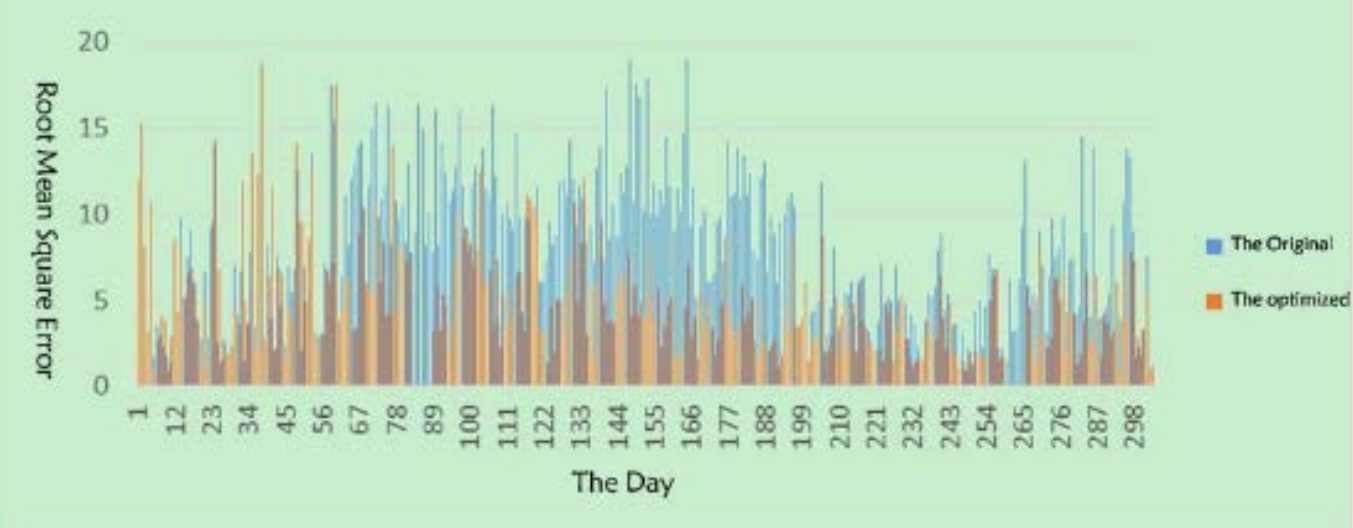

Figure 7 The Comparison of Conditions of Power Forecast before and after the Improvement in the Whole Year 2013 
It can be seen from Figure 7 that the whole root-mean-square error decreases a little after the optimization, which can mainly makes the wind power plant free from the examination of wind power prediction. The average daily accuracy rate increases from the original $84 \%$ to the present $88 \%$.

\section{Conclusion}

Through the comparison of the improved power prediction system with the original prediction system in Zhong San Jia Wind Power Plant and the error comparison distinguishing between the strong wind months and the weak wind months, it is found that the whole root-mean-square error decreases obviously in the strong winds after the improvement of the system. Meanwhile, the average daily accuracy rate increases by 6 per cent and it increases from the original $88.56 \%$ to the present $90 \%$ in the weak wind months. As a whole, the average daily accuracy rate increases from the original $84 \%$ to the present $88 \%$, which shows excellent effects of the improved system.

\section{References}

[1] National Energy Administration. Regulations on Wind Power Forecast and Its Coordinated Operation (trial implementation) [R]. Beijing: National Energy Administration, 2012.

[2]DamousisIGDokopoulosP.Afuzzyexpertsystemfortheforecastingofwindspeedandpowergeneratio ninwindfarms[C]//IEEEPowerIndustryComputerApplicationsConference, Sydney, Australia: 2001.

[3] WANG Lijie, LIAO Xiaozhong, GAO Shuang \& DONG Lei. Chaos Characteristics Analysis of Wind Power Generation Time Series for a Grid Connecting Wind Farm [J]. TRANSACTIONS OF BEIJING INSTITUTE OF TECHNOLOGY, 2007, 12.

[4] LI Zhi, HAN Xueshan, HAN Li \& KANG Kai. An Ultra-short-term Wind Power Forecasting Method in Regional Grids [J]. AUTOMATION OF ELECTRIC POWER SYSTEMS, 2010, 07.

[5] XU Man, QIAO Ying \& LU Zongxiang. A Comprehensive Error Evaluation Method for Short-term Wind Power Prediction [J]. AUTOMATION OF ELECTRIC POWER SYSTEMS, 2011, 12.

[6] LI Chuang, SHEN Zhu, MENG Kaifeng, SUN Hanmo \& YIN Shi. Modeling for power curve of load limited wind turbine base on hybrid modeling method [J]. Renewable Energy Resources, 2013,13.

[7]MILLIGAN M,SCHWARTZ M,WAN Y. Statistical wind power forecasting models:results for U.S.wind farms[A].Austin:AWEA,2003.

[8] BOONE A. Simulation of short-term wind speed forecast errors using a multi-variate arma(1,1) time-series model[D].Stockholm:Royal Institute of Technology,2005. 